\section{Kidney \\ Blood Pressure Research}

\title{
Fluorofenidone Attenuates Oxidative Stress and Renal Fibrosis in Obstructive Nephropathy via Blocking NOX2 (gp91phox) Expression and Inhibiting ERK/MAPK Signaling Pathway
}

\author{
Jiao Qin ${ }^{a} \quad$ Wen-Juan Mei ${ }^{b}$ Yan-Yun Xie ${ }^{b} \quad$ Ling Huang $^{b} \quad$ Qiong-Jing Yuan ${ }^{b}$ \\ Gao-Yun Huc Li-Jian Tao ${ }^{\text {b,d }}$ Zhang-Zhe Peng ${ }^{b}$ \\ aDivision of Nephropathy, Changsha Central Hospital; ${ }^{b}$ Division of Nephropathy, Department of \\ Medicine, Xiangya Hospital; ' Faculty of Pharmaceutical Sciences; ' ${ }^{\mathrm{S}}$ State Key Laboratory of Medical \\ Genetics of China, Central South University, Changsha, Hunan, China
}

\section{Key Words}

Fluorofenidone $•$ Obstructive nephropathy $・$ NOX2 (gp91phox) $・$ ERK • Losartan

\begin{abstract}
Background/Aims: We evaluated the therapeutic effects of fluorofenidone (AKF-PD), a novel pyridone agent, targeting oxidative stress and fibrosis in obstructive nephropathy. Methods: AKF-PD was used to treat renal interstitial fibrosis in unilateral ureteral obstruction (UUO) obstructive nephropathy in rats. The expression of NOX2 (gp91phox), fibronectin and extracellular signal regulated kinase (ERK) were detected by western blot. A level of Malondialdehyde (MDA), an oxidative stress marker, was measured by ELISA. In addition, ROS and the expressions of NOX2, collagen I (a1), fibronectin and p-ERK were measured in angiotensin (Ang) II-stimulated rat proximal tubular epithelial cells (NRK-52E) in culture. Results: In NRK-52E cells, AKF-PD reduced AngII induced expressions of ROS, NOX2, fibronectin, collagen I (a1) and p-ERK. In UUO kidney cortex, AKF-PD attenuated the degree of renal interstitial fibrosis, which was associated with reduced the expressions of collagen I (a1) and fibronectin. Furthermore, AKF-PD downregulated the expressions of NOX2, MDA and p-ERK. Conclusion: AKF-PD treatment inhibits the progression of renal interstitial fibrosis by suppressing oxidative stress and ERK/MAPK signaling pathway.
\end{abstract}




\section{Kidney Blood Pressure Research}

\section{Introduction}

Upper urinary tract obstruction is the leading cause of impaired fluid movement [1], which can give rise to renal ischemia due to intrapelvic pressure increased and renal hemodynamic changed. Moreover, it is highly possible that can eventually result in renal fibrosis and damage to renal function. Surgery can remove a urinary tract obstruction but it cannot reverse renal fibrosis, so techniques to prevent fibrosis are urgently needed.

Obstructive nephropathy is typically associated with increased generation of free radicals and/or impaired antioxidant defense, resulting in increased reactive oxygen species (ROS), which contribute to the onset, progression, and pathological consequences of obstructive nephropathy [2]. An imbalance between oxidant and antioxidant systems may cause additional injury to the interstitial tissue, and lead to renal tubular atrophy and interstitial fibrosis. Therefore, ROS generated in renal tissues and antioxidant imbalances are important pathogenic mechanisms of obstructive nephropathy. ROS are ubiquitous signaling molecules in biological systems, and the NADPH oxidase (NOX) enzyme family is an important source of ROS. Five homologues of the catalytic NOX subunit (NOX1-5) have been identified [3]. The NOX family has been linked to various cellular functions, such as proliferation, migration, hypertrophy, metalloproteinase regulation, matrix synthesis, cell senescence, and contraction [4]. NOX2, which appears to share the same overall structure with Nox4 in Nox family, is also abundant in the kidney cortex and tubular epithelial cells [5]. Furthermore, the NOX2 has been shown to be essential for angiotensin II (Ang II)-induced oxidative stress in the kidney [6]. ROS act as second messenger, linking the signal network between Ang II and mitogen-activated protein kinases (MAPKs). Studies suggest that Ang II stimulates phosphorylation and activation of extracellular signal-regulated kinase (ERK) in renal tubular cells, depending on the formation of NAD (P)H oxidase-mediated $\mathrm{O}_{2}^{-}$[7].

Fluorofenidone (1-(3-fluorophenyl)-5-methyl-2-(1H)-pyridone, AKF-PD) is a novel pyridone agent, molecular formula: $\mathrm{C} 12 \mathrm{H} 100 \mathrm{NF}$, structural formula as shown below. Several studies have reported that it can attenuate fibrosis in kidney, liver, lung and heart [8-11]. We have reported previously that AKF-PD attenuates collagen I and transforming growth factor-beta1 expression through a Nox4-dependent way in rat proximal tubular epithelial cells (NRK-52E) [9], suggesting its potential anti-oxidative property.

However, the role of AKF-PD in NOX2-depended oxidative stress and fibrosis is not well understood in a rat model of obstructive nephropathy induced by unilateral ureteral occlusion (UUO) and in Ang II-stimulated NRK-52E cells. At the same time, whether ERK/ MAPK signal pathway involves in NOX2-depended ROS and collagen expression by Ang II is unclear. Therefore, we investigated the mechanisms behind the putative antioxidant and antifibrotic effects of AKF-PD on obstructive nephropathy in rats model with UUO. We hypothesized that AKF-PD inhibits NOX2-depended ROS and collagen expression partly via ERK/MAPK signal pathway in the pathogenesis of obstructive nephropathy. Losartan, an Ang II receptor blocking agent, was used to create positive control.

\section{Materials and Methods}

\section{Materials}

All reagents for cell culture were purchased from Invitrogen (Carlsbad, CA, USA). Antibodies against p-ERK1/2, ERK1/2, MEK and U0126 were purchased from Cell signaling Inc. (Danvers, Mass, MA), collagen I (a1) and Fibronectin were purchased from Santa Cruz Biotechnology (Santa Cruz, CA,Calif,USA), and NOX2 (gp91phox) was purchased from Abcam (Cambridge, UK). Anti- $\beta$-actin and a cytochrome c reductase (NADPH) assay kit were purchased from Sigma-Aldrich Ltd. (St. Louis, MO, USA). Dichlorodihydrofluorescein diacetate (DCFH-DA) was purchased from Invitrogen and the Oxiselect ${ }^{\mathrm{TM}}$ thiobarbituric acid reactive substances (TBARS) assay kit for malondialdehyde (MDA) quantification was purchased from Cell Biolabs, Inc. (San Diego, CA, USA). 


\section{Kidney \\ Blood Pressure Research}

Kidney Blood Press Res 2015;40:89-99

\begin{tabular}{l|l}
\hline DOI: $10.1159 / 000368485$ & C) 2015 S. Karger AG, Basel \\
Publisned ontIne: IVIarch 05, 2015 & www.karger.com/kbr
\end{tabular}

Qin/Mei/Xie et al.: Fluorofenidone Attenuates Oxidative Stress and Renal Fibrosis

The HRP-conjugated secondary antibodies and enhanced chemiluminescence (ECL) kit for Western blot were purchased from Amersham Biosciences (Piscataway, NJ). AKF-PD (Lot No.090601) was synthesized by Sunshine Lake Pharma Co, Ltd. (Shenzhen, Guangdong, China). Losartan was from Merck \& Co, Inc (Whitehouse Station, NJ). All other chemicals were from Sigma-Aldrich Ltd unless otherwise indicated.

\section{Cell Culture and Treatment}

Rat proximal-tubular epithelial (NRK-52E) cells were purchased from ATCC (Rockville, MD). NRK-52E cells were cultured in DMEM supplemented with $8 \% \mathrm{FBS}$, penicillin (100 U/ml) and $100 \mu \mathrm{g} / \mathrm{ml}$ streptomycin (Invitrogen), at $37^{\circ} \mathrm{C}$ in a humidified atmosphere of $5 \% \mathrm{CO}_{2}$ and $95 \%$ air. The cells were seeded on six-well culture plates to $60-70 \%$ confluence in complete medium containing $5 \%$ FBS for $24 \mathrm{~h}$, which was changed to serum-free media for $24 \mathrm{~h}$ before Ang II induction at a final concentration of $10^{-7} \mathrm{~mol}$. To examine the effects of AKF-PD and losartan on Ang II-induced expression of p-ERK, NOX2, the cells were treated with AKF-PD ( $2 \mathrm{mM})$ or losartan $\left(10^{-5} \mathrm{~mol}\right)$ for $24 \mathrm{~h}$, were incubated with the MEK inhibitor U0126 $(10 \mu \mathrm{M}$, Cell Signaling) or NADPH oxidase inhibitor DPI $\left(10^{-7} \mathrm{~mol}\right.$, Sigma) for $1 \mathrm{~h}$, and were subsequently induced by Ang II $\left(10^{-7} \mathrm{~mol}\right)$ for $15 \mathrm{~min}$ (p-ERK) and $24 \mathrm{~h}$ (NOX2, fibronectin) before cellular protein extraction. Each experiment was replicated three times.

\section{Transient Transfection Assays}

Plasmids constitutively expressing the active form of MEK (MEK1Q56P) were kind gifts from Dr. Damu Tang (McMaster, CA). Transient transfection of cells with one or the other plasmid was conducted using Lipofectamine 2000 reagent (Invitrogen Corporation, Carlsbad, CA, USA) following the protocol provided by the manufacturer. The production of $\mathrm{O}_{2}^{-}$was then measured in these groups of cells (see below) following treatment with AKF-PD for $24 \mathrm{~h}$. Each experiment was carried in triplicate.

\section{Measurement of $\mathrm{O}_{2}^{-}$Generation in Intact Cells}

Changes in intracellular ROS levels were measured by quantifying the oxidative conversion of cellpermeable 2', 7'-dichlorofluorescein diacetate (DCFH-DA) to fluorescent dichlorofluorescein (DCF) using a Microplate Reader (BioTek Inc, Winooski, VT). In brief, cells were seeded onto a 96-well plate and treated as described above. Cells were then washed with D-Hank's solution and incubated with DCFH-DA (10 $\mu \mathrm{M})$ at $37^{\circ} \mathrm{C}$ for $20 \mathrm{~min}$ in the dark. Cells were then washed in PBS, trypsinized, and resuspended in $100 \mu \mathrm{l}$ of PBS. Cellular ROS resulting from the oxidation of 2', 7'-dihydrofluorescein was measured (excitation: 488 $\mathrm{nm}$; emission: 515 to $540 \mathrm{~nm}$ ). Fluorescence data are expressed as percent increase over untreated samples.

In addition, transfected NRK-52E cells were seeded onto a 50-mm dish and treated as described above. After washing with PBS solution, cells were incubated with DCFH-DA $(10 \mathrm{mM})$ at $37^{\circ} \mathrm{C}$ for 20 min in the dark. The oxidative conversion of cell-permeable DCFH-DA to fluorescent DCF was assessed by flow cytometry using FACS Calibur (Becton Dickinson, Franklin Lakes, NJ). The percentage of fluorescent intensity was assessed using ModFit LT software (Verity Software House, Topsham, ME). Fluorescence data are expressed as percent increase over untreated samples. Each experiment was performed in triplicate.

\section{Animals and Treatments}

A total of 24 male Sprague-Dawley (SD) rats (220-250 g) were randomly assigned to four groups (N = 6) as follows: (1) UUO rats treated with $0.5 \%$ carboxymethyl cellulose sodium (CMC-Na) (designated as UUO); (2) UUO rats treated with $500 \mathrm{mg} / \mathrm{kg}$ per day AKF-PD (UUO + AKF-PD); (3) UUO rats treated with $20 \mathrm{mg} / \mathrm{kg}$ per day losartan (UUO + losartan); and (4) sham rats treated with $0.5 \%$ CMC-Na (sham). All rats were killed at day 14 after surgery, and the left kidney was decapsulated. UUO operation method reference to Le Tulzo Y SR et al. [12].

\section{Histopathological Evaluation}

Kidney tissue sections were deparaffinized in xylene, rehydrated through graded ethanol, and subsequently stained with hematoxylin and eosin (H\&E) and Masson's trichrome, or set aside for immunohistochemistry. The tubulo-interstitial damage index was graded as described previously [13]. Scores were determined in 20 randomly-selected fields per section examined under $200 \mathrm{x}$ magnifications. In Masson's trichrome stained samples, tubulo-interstitial fibrosis was estimated using the following scoring 


\section{Kidney \\ Blood Pressure Research}

Kidney Blood Press Res 2015;40:89-99

\begin{tabular}{l|l}
\hline DOI: $10.1159 / 000368485$ & C) 2015 S. Karger AG, Basel \\
Publisned ontIne: IVIarch 05, 2015 & www.karger.com/kbr
\end{tabular}

Qin/Mei/Xie et al.: Fluorofenidone Attenuates Oxidative Stress and Renal Fibrosis

system: $0=$ normal interstitium; $1=<25 \%$ of the surface area staining positive; $2=25-50 \%$ of the surface area staining positive ; $3=50-75 \%$ of the surface area staining positive; and, $4=>75 \%$ of the surface area staining positive.

\section{NADPH Oxidase Assay}

Renal cortex homogenate was prepared in $500 \mu \mathrm{l}$ and $1 \mathrm{ml}$ aliquots of lysis buffer by using a Dounce homogenizer (100 strokes on ice). Homogenates were then centrifuged ata low speed $(800 \mathrm{xg}, 10 \mathrm{~min})$ at $4^{\circ} \mathrm{C}$ to remove unbroken cells and debris, and aliquots were used immediately. To start the assay, $100 \mu$ l of homogenate wasadded to $900 \mu$ lof $50 \mathrm{mM}$ phosphatebuffer, $\mathrm{pH} 7.0$, containing1 mMEGTA, $150 \mathrm{mM}$ sucrose, $5 \mu$ Mlucigenin, and $100 \mu \mathrm{M}$ NADPH. Photon emission (relative light unit) was measured every 20 or $30 \mathrm{~s}$ for $10 \mathrm{~min}$ in a luminometer. There was $\mathrm{n}$ o measurable activity in the absence of NADPH. A buffer blank (less than 5\% of the cell signal) was subtracted from each reading. Superoxide production was expressed as relative chemiluminescence (light) units (RLU)/g of tissue. Protein content was measured using the Bio-Rad protein assay reagent (Hemel, Herts, UK).

\section{Measurement of Renal MDA}

Kidney tissuewashomogenizedinaqueous $0.15 \mathrm{M} \mathrm{KCl}$ solutiontoyield a $10 \%$ (v/v)homogenate. Oneml of homogenate was then mixed with one ml of $10 \%(\mathrm{v} / \mathrm{v})$ trichloroaceticacid(TCA) and centrifuged at $1,000 \mathrm{xg}$ for $15 \mathrm{~min}$. One $\mathrm{ml}$ of supernatant was suspended into $1 \mathrm{ml}$ of $0.67 \%$ 2-TBA (v/v). Sample tubes were then placed into a boiling water bath for $15 \mathrm{~min}$. Samples were allowed to cool at room temperature followed by centrifugation at $1,000 \mathrm{~g}$ for $15 \mathrm{~min}$. The optical density of the supernatant was measured at $532 \mathrm{~nm}$ using a Spectra MAX microplate reader (Molecular Devices, Sunnyvale, CA). Protein content was measured using the Bio-Rad protein assay reagent $(\mu \mathrm{mol} / \mathrm{l})$.

Western Blotting

Total $(30 \mu \mathrm{g})$ protein were extracted from fresh kidney tissue or cultured cells were separated on $10 \%$ SDS-PAGE under reducing conditions and transferred onto polyvinylidene difluoride membranes (Millipore, Bedford, MA). The unspecific binding sites were blocked in TBS-T buffer (10 mM Tris/HCl, $150 \mathrm{mM} \mathrm{NaCl}$, $0.1 \%(\mathrm{v} / \mathrm{v}$ ) Tween 20, pH 7.6) containing 5\% (w/v) skim milk for $1 \mathrm{~h}$ at room temperature. The membranes were incubated overnight at $4{ }^{\circ} \mathrm{C}$ with the primary antibodies against

NOX2 (1:1,000), Fibronectin (1:400), ERK (1:1,000), p-ERK (1:1,000) and were subsequently hybridized with HRP-conjugated secondary antibodies for $1 \mathrm{~h}$ at room temperature. The bands were visualized with an ECL kit and quantified using Glyko Bandscan 5.0 software (Glyko, Novato, CA). Each experiment was repeated three times.

\section{Statistical Analysis}

All data were expressed as means \pm standard deviation (SD). Statistical analysis was performed using SPSS 16.0 software (SPSS Inc, Chicago, IL). The comparison among different groups was made with one-way ANOVA assay. Multiple comparison tests were applied only when a significant difference was determined by ANOVA. $P<0.05$ was considered to be statistically significant.

\section{Results}

\section{AKF-PD inhibited ROS formation in Ang II-induced NRK-52E cells}

To elucidate the time course of ROS generation by Ang II stimulated rat NRK-52E cells, DCF-emitted fluorescence was measured (Fig. 1 B). Ang II-induced ROS generation was increased at $60 \mathrm{~min}$. Then, groups of $24 \mathrm{~h}$ serum-starved cells were treated with Ang II plus AKF-PD, losartan or DPI and DCF fluorescence was measured 60 min later. AKF-PD, losartan and DPI all attenuated the generation of ROS $(P<0.01)$, respectively. There was no significant difference between AKF-PD and Losartan treatment $(P>0.05)$ (Fig. 1 A, C). 


\section{Kidney Blood Pressure Research}

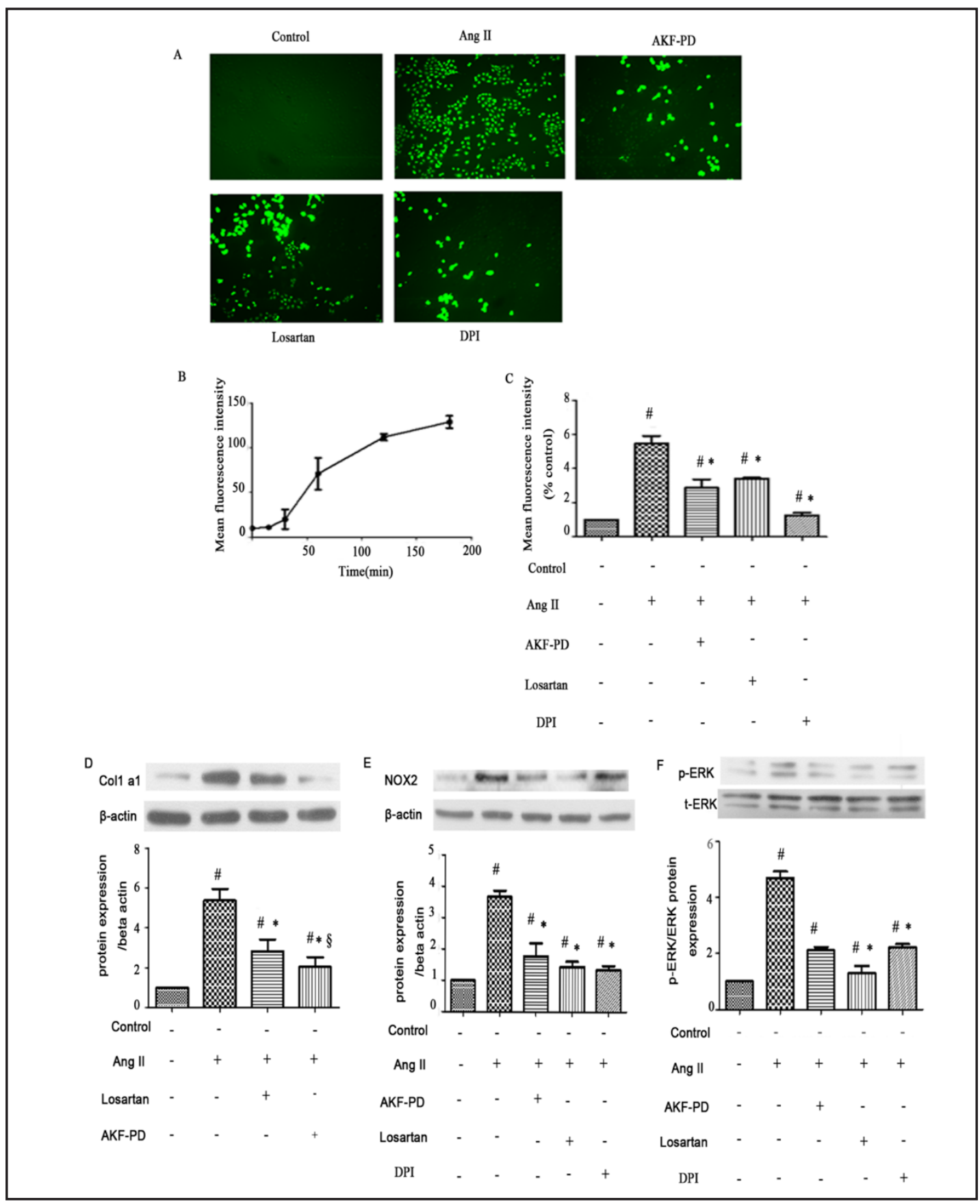

Fig. 1. The effect of AKF-PD on the Ang II-induced ROS, the expression of NOX2, and the ERK/MAPK pathway in NRK-52E cells. As shown by the conversion of cell-permeable DCFH-DA to fluorescent DCF (DCF Ang II increased DCF-sensitive cellular $\mathrm{O}_{2}^{-}$starting at $15 \mathrm{~min}$ and up to $240 \mathrm{~min}$, peaking at $60 \mathrm{~min}$ (B). Treatment with AKF-PD, $8 \times 10^{-3} \mathrm{~mol}$, losartan, $10^{-5} \mathrm{~mol}$ and NADPH oxidase inhibitor DPI $\left(10^{-7} \mathrm{~mol}\right)$ decreased ROS $(A, C)$; Western blots for Collagen I a1(D)and NOX2 (E) expression in Ang II-treated NRK52E cells with and without the addition of AKF-PD $\left(8 \times^{10-3} \mathrm{~mol}\right)$, losartan $\left(10^{-5} \mathrm{~mol}\right)$, or DPI $\left(10^{-7} \mathrm{~mol}\right)$; $\beta$-actin was used as an internal loading control, $\# P<0.05$ compared to the control group; ${ }^{*} P<0.05$ compared to the Ang II group; $§ P$ $<0.05$ compared to the losartan group. Treatment with AKF-PD, losartan, and the MEK inhibitor U0126 (10 $\mu \mathrm{M}$ ) attenuated Ang II -induced activation of p-ERK (F) for 15min in NRK-52E cells; total ERK was used as the internal loading controls. The bar graphs represent the optical density (OD) of the bands obtained by Western blotting; $\# P<0.05$ compared to the control group; ${ }^{*} P<0.05$ compared to the Ang II groups. 


\section{Kidney \\ Blood Pressure \\ Research}

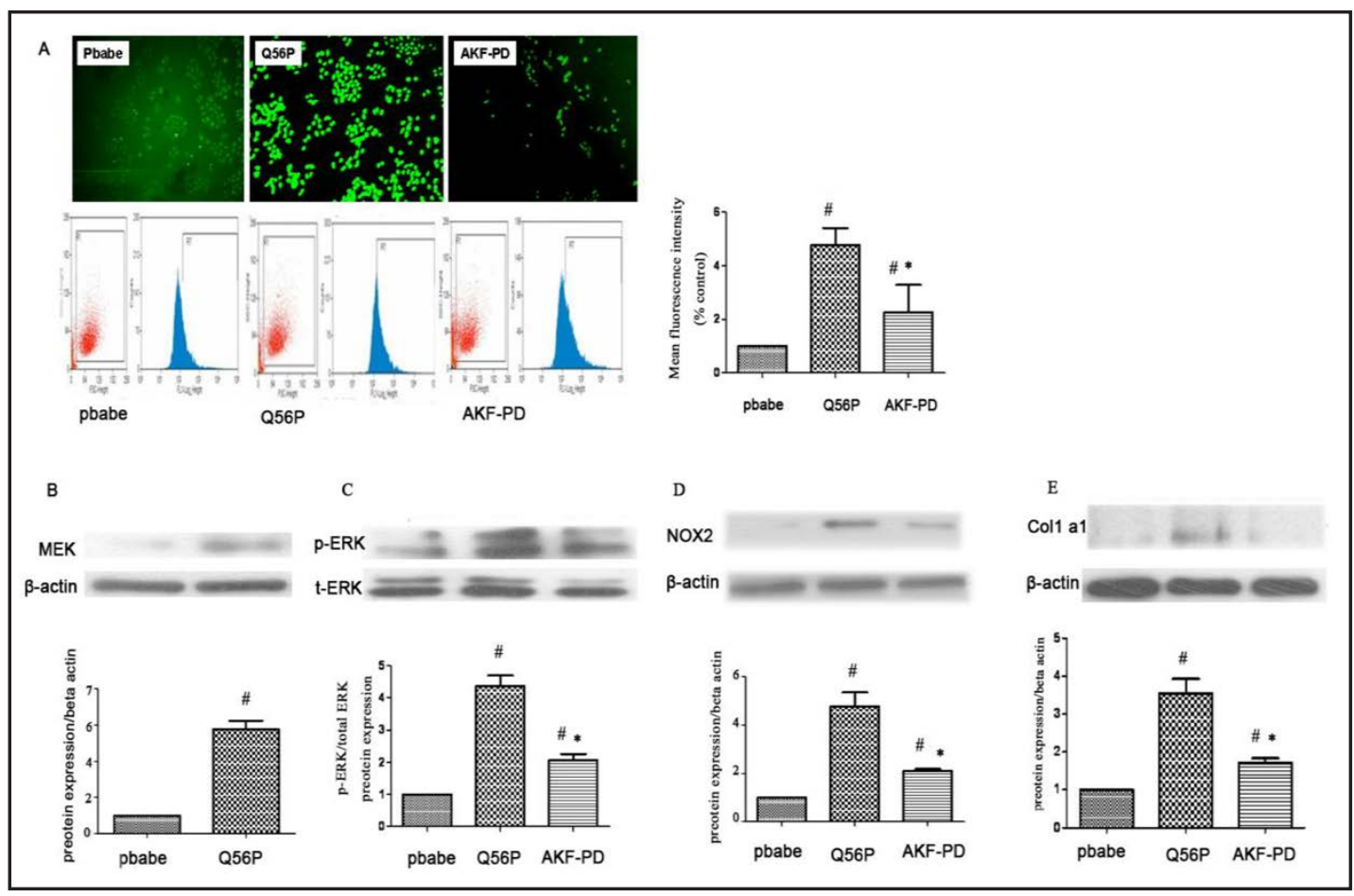

Fig. 2. Effect of AKF-PD treatment on NRK-52E cells transected with plasmids constitutively expressing the active form of MEK (MEK1Q56P). AKF-PD reduced ROS generation in cells over-expressing MEK (A). \# and * represent $P<0.01$, compared with control and MEK groups, respectively. As expected, transfection increased MEK expression (B). Bar graphs represent band OD obtained by Western blotting; \# represents $P<0.01$, compared to the MEK group. The mechanism of action of AKF-PD in NRK-52E cells overexpressing MEK (C, D, E). AKF-PD down-regulated expression of p-ERK, NOX2 and col I a1 in cells over-expressing MEK. The bar graphs represent the OD of the bands obtained by Western blotting; \# and * represent $P<0.01$, compared with the control and MEK group, respectively.

\section{AKF-PD inhibited Ang II-induced collagen I $\alpha 1$ expression in NRK-52E cells}

Ang II induced significantly more collagen I $\alpha 1$ expression compared to the control group (Fig. 1D) $(P<0.01)$. Either AKF-PD or losartan pretreatment for $24 \mathrm{~h}$ significantly blocked Ang II-induced collagen I $\alpha 1$ expression $(P<0.01)$. The effect of AKF-PD was better than losartan in Ang II induced collagen I $\alpha 1$ expression $(P<0.01)$.

AKF-PD inhibited expression of NOX2 in Ang II-induced NRK-52E cells

Cells pretreated (24 h) with Ang II alone increased the expression of NOX2 $(P<0.01)$ (Fig. $1 \mathrm{E})$. Any pretreatment with AKF-PD, losartan for $24 \mathrm{~h}$, DPI for $1 \mathrm{~h}$ significantly inhibited Ang II-induced the expression of NOX2 $(P<0.01)$. There was no significant difference between AKF-PD and Losartan treatment $(P>0.05)$.

AKF-PD inhibited ERK/MAPK signaling pathway in Ang II-induced NRK-52E cells

To further decipher the molecular mechanisms responsible for the inhibition of AKFPD on Ang II-induced NOX2 activation, we investigated the ERK/MAPK signaling pathway in NRK-52E cells. As shown in (Fig. $1 \mathrm{~F}$ ), a 15 min Ang II treatment induced significantly increased level of or concentration of phosphorylated ERK in NRK-52E cells. Any pretreatment with AKF-PD, losartan for $24 \mathrm{~h}$, MEK inhibitor of U0126 for $1 \mathrm{~h}$ significantly inhibited the expression of p-ERK. There was no significant difference between AKF-PD and Losartan treatment $(P>0.05)$. 


\section{Kidney Blood Pressure Research}

Fig. 3. Histological evaluation of renal cortex samples obtained from rats treated for 14 days with AKF-PD or losartan after surgically induced UUO. Sham: the ureter was surgically manipulated but not ligated; UUO +AKFPD: UUO rats treated with $500 \mathrm{mg} /$ kg/day AKF-PD; UUO + losartan; UOO rats treated with $20 / \mathrm{mg} / \mathrm{kg} /$ day losartan; $n=6$ per group. (A) H\&E stained samples; (B) Masson's trichrome stained samples. $\# P<0.05$ compared to the Sham group; ${ }^{*} P<0.05$ compared to the UUO group. (E)Gels are representative from Western blot analysis for fibronectin (FN) protein expression in renal cortex cells from Sham,

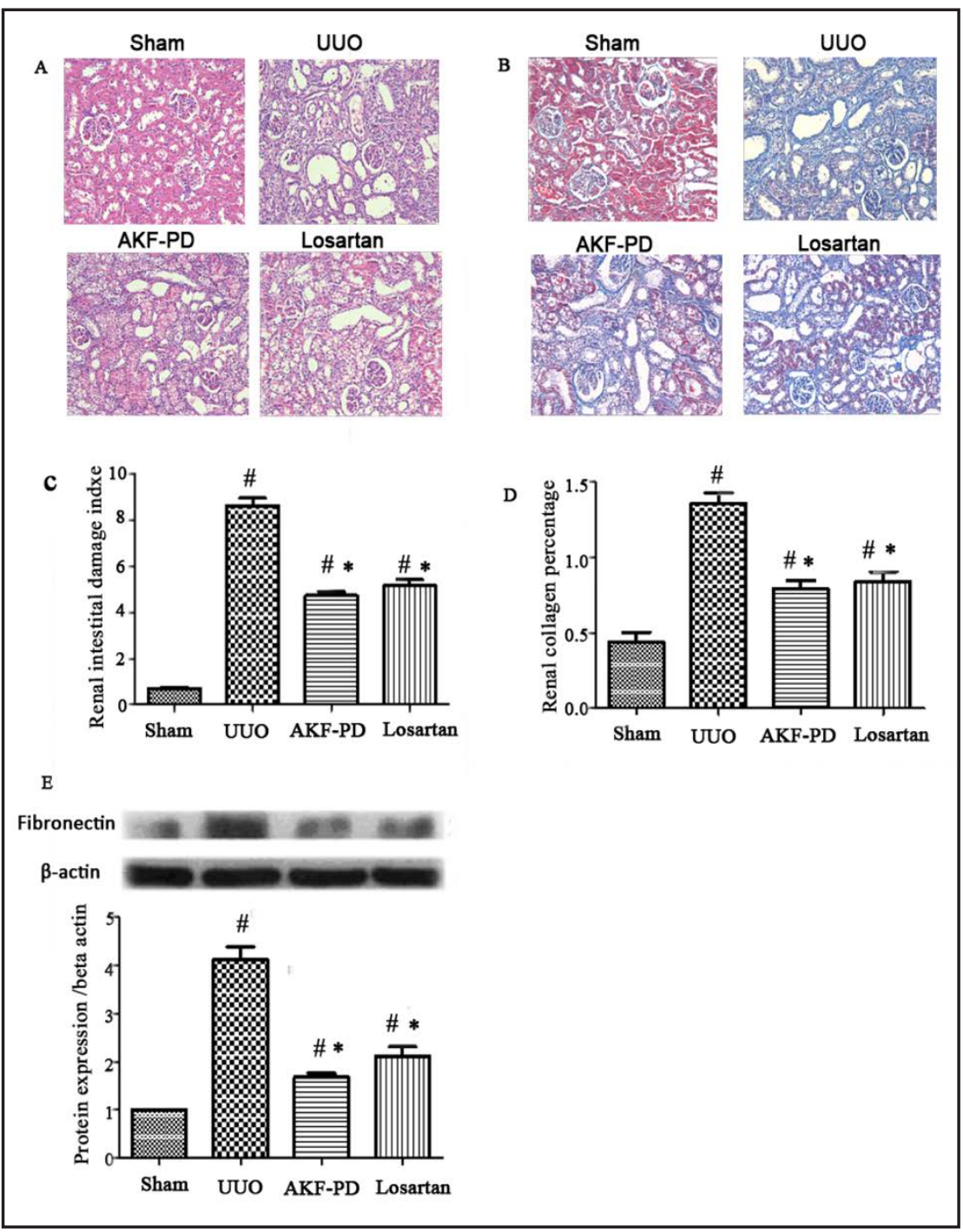
UUO, UUO +AKF-PD

rats, UUO + losartan rats; $\beta$-actin was the internal loading control. Bar graphs represent the optical density (OD) of the bands resulting from Western blot for relative quantification of protein. $\# P<0.05$ compared to the Sham group; ${ }^{*} P<0.05$ compared to the UUO group; $\mathrm{n}=6$ per group.

AKF-PD inhibited expression of NOX2, collagen I $\alpha 1$ and generation of ROS in NRK-52E cells via ERK/MAPK signaling pathway

As shown in Fig. 2, NRK-52E cells were transfected with MEK1Q56P plasmids (Fig. 2 B). After transient transfection, NRK-52E cells overexpressing MEK have elevated generation of ROS (Fig. 2 A) and expression of p-ERK (Fig. 2 C), NOX2 (Fig. 2 D), collagen I $\alpha 1$ (Fig. 2 E) However, the addition of AKF-PD dramatically reduced the amount of p-ERK, NOX2 and collagen I $\alpha 1$.

AKF-PD attenuated histological injures and ECM deposition in UUO rats

As show in (Fig. $3 \mathrm{~A}$ ), rats with UUO exhibited kidney damage which was tubulo-interstitial injury consisting of tubular atrophy, interstitial infiltration of fibroblasts, interstitial edema, the presence of red cell and protein casts, and renal tubular epithelial cell vacuolar degeneration. 


\section{Kidney \\ Blood Pressure \\ Research}

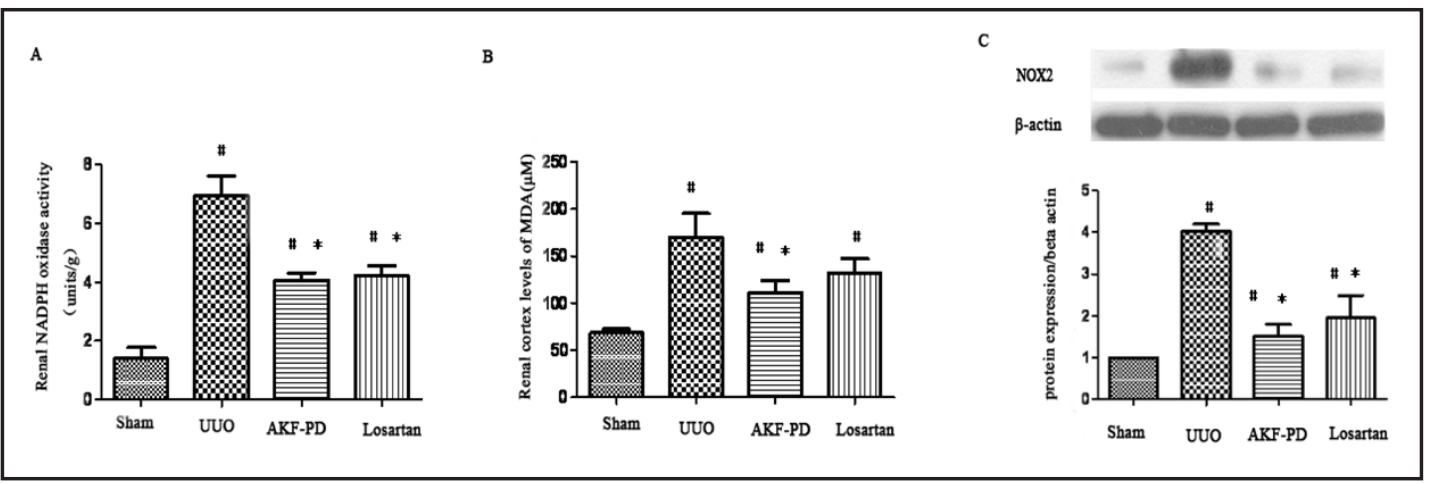

Fig. 4. Oxidative activity in renal cortex samples obtained from rats after surgically induced UUO. Sham operated rats underwent ureteral manipulation without ligation. Groups of UUO rats were treated with $500 \mathrm{mg} / \mathrm{kg} /$ day AKF-PD or $20 / \mathrm{mg} / \mathrm{kg} /$ day losartan for 14 days. Treatment with AKF-PD decreased NADPH oxidase activity (A), and MDA(B), but only AKF-PD decreased MDA in renal cortex tissue from UUO rats (B). Gels are representative from Western blot analysis for: NOX2 (C) protein expression in renal cortex cells from Sham, UUO, UUO +AKF-PD rats; $\beta$-actin was the internal loading control. Bar graphs represent the optical density (OD) of the bands resulting from Western blot for relative quantification of protein. $\# P<0.05$ compared to the Sham group; ${ }^{*} P<0.05$ compared to the UUO group; $\mathrm{n}=6$ per group.

AdministrationofbothAKF-PDandlosartansignificantlyattenuatedrenalpathologicalalterations and tubulointerstitial damage as evidenced by downregulating renal interstitial damage indexes $(P<0.05)$ (Fig. 3 C).

While the interstitial accumulation of collagen fibers were increased in the kidneys from UUO rats, both AKF-PD and losartan treatment attenuated $(P<0.05)$ collagen expression (Fig. $3 \mathrm{~B}, \mathrm{D}$ ). In support of ECM remodeling, UUO rat kidneys had increased fibronectin expression compared to sham-operated rats (Fig. 3 E). Furthermore, AKF-PD and losartan treatment ameliorated $(P<0.05)$ increased fibronectin expression observed in UUO rats. There was no statistical difference in renal fibronectin expression between AKF-PD and losartan-treated rats.

\section{AKF-PD attenuated NOX enzyme activity and expression of NOX2 in UUO rats}

Kidneys from UUO rats underwent oxidative stress as evidenced by significantly increased NOX2 protein expression and NOX enzyme activity $(P<0.05)$. AKF-PD and losartan treatment attenuated NOX2 expression and NOX enzyme activity $(P<0.05)$, There was no statistical difference between AKF-PD and losartan treatment $(P<0.05)$ (Fig.4 C, A).

\section{$A K F-P D$ attenuated lipid peroxidation in UUO rats}

Kidneys from UUO rats underwent oxidative stress as evidenced by significantly increased MDA of lipid peroxidation. AKF-PD decreased MDA level $(P<0.05)$ and there was no statistical difference between AKF-PD and losartan treatment groups (Fig. 4 B).

\section{Discussion}

Antioxidant strategies have offered important preventive and therapeutic improvement in the development of renal injury, both in vitro and in vivo [12], so we investigated the potential effects of fluorofenidone (AKF-PD) upon oxidative stress-driven kidney damage in UUO models. We revealed that AKF-PD had an obvious renoprotective effect as evidenced by decreased histological injures and ECM deposition in UUO rats. These observations are 
in accordance with fluorofenidone reducing NOX2 expression and MDA level in 14 days UUO rats, an indicator of oxidative stress. Collectively, our data show that AKF-PD exerts its renoprotective role by decreasing oxidative stress and ECM deposition in UUO rats.

UUO animal models are appropriate for studying mechanisms of human obstructive nephropathy as well as the impact of potential therapeutic approaches to ameliorate renal disease. Obstructive nephropathy is characterized by oxidative stress promoted by cytokines and growth factors produced by damaged tubular cells and accumulated myofibroblasts. This oxidative stress contributes to tubular atrophy and interstitial fibrotic characteristics of obstructive nephropathy [13]. Reactive oxygen species are chemically reactive molecules and free radicals generated from molecular oxygen that, if produced in excess, cause damage to tissues and different components of the cells. The production of ROS by renal tubular epithelial cells is considered to be closely associated with the pathogenesis of UUO-induced renal fibrosis. Although mitochondrial respiration generated greater $\mathrm{O}_{2}^{-}, \mathrm{NAD}(\mathrm{P}) \mathrm{H}$ oxidase is known to be the principal source of abnormal cellular signaling [14]. NAD(P)H oxidase was originally found in neutrophils and is composed of the catalytic subunit gp91phox together with the regulatory subunits p22phox, p47phox, and p67phox and the small GTPase Rac [15]. Electrons from $\mathrm{NAD}(\mathrm{P}) \mathrm{H}$ are transferred through the enzyme to molecular oxygen to generate superoxide and subsequently other ROS such as hydrogen peroxide. Regarding mechanisms leading to oxidative stress, NOX2 is a major source of vascular and tissue $\mathrm{O}_{2}$ anion formation that is expressed in normal rat kidney [16]. Moreover, as the most abundant subunit of the NOX family in the kidney, NOX2 is a major source of ROS and functions as an oxygen sensor regulating oxygen-dependent gene during renal inflammatory processes [17]. We have proven that NOX2 protein expression in obstructive renal interstitium is increased concomitantly with elevated MDA 14 days after UUO and with increased ROS in NRK-52E cells induced by Ang II.

Searching for novel agents with inhibitory effects on NOX activation is attracting increasing attention for the prevention of renal fibrosis. A vicious cycle of activation of the renal angiotensin-aldosterone system and ROS plays a pivotal role in the progression of renal injury [18]. We hypothesized that AKF-PD-driven blockage of oxidative stress in renal tubular epithelial cells was likely mediated by antagonizing Ang II signaling. Taken together, the above observations suggest the notion that AKF-PD offers a better renoprotection in part via more effectivetly attenuating the ERK-NOX2 pathway.

Many signaling pathways can regulate NOX2 expression, such as MAPK, TGF- $\beta 1 / \mathrm{Smad}$ and PI3K/Akt/ROS $[15,19]$. Some reports suggest that Ang II stimulated mitochondrial ROS generation, through the opening of mitochondrial KATP channels leading to redox sensitive activation of MAPK [20]. NADPH oxidase-mediated ROS and subsequent ERK and p38 MAPK activation (ROS-MAPK pathways) play important roles in TGF- $\beta 1$-induced ECM accumulation in renal tubular epithelial cells [21]. Our results suggest that NADPH oxidasemediated ROS depending on ERK activation (ERK-ROS pathway) also play important roles in ECM accumulation in renal interstitium. We assume that there's a strong positive feedback loop between ERK signals and ROS that would further aggravate renal interstitial fibrosis.

ERKactivation plays important roles in ECM accumulation in the renal tubulointerstitium. ERK signaling pathway is required for both the restoration of damaged tubular epithelial cells and the inhibition of fibrosis progression following injury [22]. In this study, we demonstrate that the renoprotective effect of AKF-PD might depend on reducing ERK activation, which aligns with findings in mouse mesangial cells and renal interstitial fibroblasts $[23,24]$.We assume that the antifibrotic and antioxidative effects of AKF-PD are suggested to be mediated by its actions through inhibition of ERK activation.

Losartan, an angiotensin II type 1 (AT1) receptor antagonist selected in the present study as the positive control drug, has significant antifibrotic effects on non-diabetic and diabetic nephropathy [25]. Losartan was also reported to be able to attenuate oxidative stress [26]. Our data demonstrated the anti-oxidative stress effects of Losartn via blunting the attenuated NOX enzyme activity and expression of NOX2 in UUO rats and NRK-52E 


\section{Kidney \\ Blood Pressure Research}

cells, preventing ECM degradation, which were previously reported by our group [2], More importantly, this study suggested that AKF-PD not only prevented tubulointerstitial fibrosis and oxidative stress in the UUO rat model, but also exerted some more effective anti-fibrosis and ant-oxidative stress abilities than Losartan regarding to some important renal fibrosis and Lipid peroxidat indices, including MDA production, collagen1a1 protein levels. At the same time we confirmed that AKF-PD did not influence body weight, uric acid and serum creatinine in AKF-PD treated rats compared to UUO rats, which was in accordance with a previous study [23], these data lead to more advantages of AKF-PD than that of Losartan in the improvement of obstructive nephropathy, to some extent.

\section{Conclusion}

It is also possible that AKF-PD derived renoprotection may not be limited to in obstructive nephropathy. Evidence supports a pathological role of PKC-p22phox (NADPH oxidative) in developing diabetic nephropathy [27]. AKF-PD may also display a protective capacity for these obstructive injuries. These effects are possibly mediated by attenuation of NOX2-depended oxidative stress, ERK phosphorylation and ECM accumulation. ERK pathway may involve in NOX2 depended ROS and collagen expression induced by Ang II in NRK-52E cells. AKF-PD inhibits oxidative stress and fibrosis at least partly via the ERK/ MAPK signaling pathway. It is hoped that this information will lend insight into the complex issues surrounding the use of AKF-PD in obstructive nephropathy.

\section{Disclosure Statement}

All authors declare that they have no competing financial interests.

\section{Acknowledgements}

This research was supported by the grants from the National Natural Science Foundation of China (No. 81001467, No. 11JJ2051, No.81400749).

\section{References}

1 Klahr S, Morrissey J: Obstructive nephropathy and renal fibrosis. Am J Physiol Renal Physiol 2002;283:F861-F875.

-2 Qin J, Xie YY, Huang L, Yuan QJ, Mei WJ, Yuan XN, Hu GY, Cheng GJ, Tao LJ, Peng ZZ: Fluorofenidone inhibits nicotinamide adeninedinucleotide phosphate oxidase via PI3K/Akt pathway in the pathogenesis of renal interstitial fibrosis. Nephrology (Carlton) 2013;18:690-699.

-3 Dendooven A, Ishola Jr. DA, Nguyen TQ, Van der Giezen DM, Kok RJ, Goldschmeding R, Joles JA: Oxidative stress in obstructive nephropathy. Int J Exp Path 2011;92:202-210.

-4 Touyz RM, Briones AM, Sedeek M, Burger D, Montezano AC: NOX Isoforms and Reactive Oxygen Species in Vascular Health. Mol Interv 2011;11:27-35.

-5 Wolak T, Kim HJ, Ren Y, Kim J, Vaziri ND, Nicholas SB: Osteopontin modulates angiotensin II-induced inflammation, oxidative stress, and fibrosis of the kidney. Kidney Int 2009;76:32-43.

-6 Garrido AM, Griendling KK: NADPH oxidases and angiotensin II receptor signaling. Mol Cell Endocrinol 2009;302:148-158.

7 Sachse A, Wolf G: Angiotensin II-Induced Reactive Oxygen Species and the Kidney. J Am Soc Nephrol 2007;18:2439-2446. 


\section{Kidney \\ Blood Pressure Research}

Kidney Blood Press Res 2015;40:89-99

DOI: 10.1159/000368485

Publisned ontıne: vararch 05, 2015

(C) 2015 S. Karger AG, Basel

www.karger.com/kbr

8 Meng JZ, Hu C, Zhu Y, Peng Z, Hu G, Wang Z, Tao L: Fluorofenidone attenuates bleomycin-induced pulmonary inflammation and fibrosis in mice via restoring caveolin 1 expression and inhibiting mitogenactivated protein kinase signaling pathway. Shock 2012;38:567-573.

-9 Peng ZZ, Hu GY, Shen H, Wang L, Ning WB, Xie YY, Wang NS, Li BX, Tang YT, Tao LJ: Fluorofenidone attenuates collagen I and transforming growth factor-beta1 expression through a nicotinamide adenine dinucleotide phosphate oxidase-dependent way in NRK-52E cells. Nephrology (Carlton) 2009;14:565-572.

10 Chen LX, Yang K, Sun M, Chen Q, Wang ZH, Hu GY, Tao LJ: Fluorofenidone inhibits transforming growth factor-beta1-induced cardiac myofibroblast differentiation. Pharmazie 2012;67:452-456.

11 Peng Y, Yang H, Zhu T, Zhao M, Deng Y: The antihepatic fibrotic effects of fluorofenidone via MAPK signalling pathways. Eur J Clin Invest 2013;43:358-368.

12 Forbes JM, Coughlan MT, Cooper ME: Oxidative Stress as a Major Culprit in Kidney Disease in Diabetes. Diabetes 2008;57:1446-1454.

-13 Moriyama T, Kawada N, Nagatoya K, Takeji M, Horio M, Ando A, Imai E, Hori M: Fluvastatin suppresses oxidative stress and fibrosis in the interstitium of mouse kidneys with unilateral ureteral obstruction. Kidney Int 2001;59:2095-2103.

$\checkmark 14$ Ozbek E, Ilbey YO, Ozbek M, Simsek A, Cekmen M, Somay A: Melatonin Attenuates Unilateral Ureteral Obstruction-Induced Renal Injury by Reducing Oxidative Stress, iNOS, MAPK, and NF-kB Expression. J Endourol 2009;23:1165-1173.

15 Kimura S, Zhang GX, Nishiyama A, Shokoji T, Yao L, Fan YY, Rahman M, Suzuki T, Maeta H, Abe Y: Role of $\mathrm{NAD}(\mathrm{P}) \mathrm{H}$ oxidase- and mitochondria-derived reactive oxygen species in cardioprotection of ischemic reperfusion injury by angiotensin II. Hypertension 2005;45:860-866.

16 Singh U, Jialal I: Oxidative stress and atherosclerosis. Pathophysiology 2006;13:129-142.

17 Geiszt M, Kopp JB, Várnai P, Leto TL: Identification of Renox, an NAD(P)H oxidase in kidney. Proc Natl Acad Sci USA 2000;97:8010-8014.

18 Folli F, Corradi D, Fanti P, Davalli A, Paez A, Giaccari A, Perego C, Muscogiuri G: The Role of Oxidative Stress in the Pathogenesis of Type 2 Diabetes Mellitus Micro- and Macrovascular Complications: Avenues for a Mechanistic-Based Therapeutic Approach. Curr Diabetes Rev 2011;7:313-324.

19 Bondi CD, Manickam N, Lee DY, Block K, Gorin Y, Abboud HE, Barnes JL: NAD(P)H Oxidase Mediates TGF-ß1-Induced Activation of Kidney Myofibroblasts. J Am Soc Nephrol 2010;21:93-102.

-20 Zhang GX, Lu XM, Kimura S, Nishiyama A: Role of mitochondria in angiotensin II-induced reactive oxygen species and mitogen-activated protein kinase activation. Cardiovasc Res 2007;76:204-212.

21 Rhyu DY, Park J, Sharma BR, Ha H: Role of reactive oxygen species in transforming growth factor-beta1induced extracellular matrix accumulation in renal tubular epithelial cells. Transplant Proc 2012;44:625628.

22 Okada H, Kalluri R: Cellular and Molecular Pathways that Lead to Progression and Regression of Renal Fibrogenesis. Curr Mol Med 2005;5:467-474.

-23 Yuan Q, Wang R, Peng Y, Fu X, Wang W, Wang L, Zhang F, Peng Z, Ning W, Hu G, Wang Z, Tao L: Fluorofenidone attenuates tubulointerstitial fibrosis by inhibiting TGF- $\beta(1)$-induced fibroblast activation. Am J Nephrol 2011;34:181-194.

-24 Wang L, Hu GY, Shen H, Peng ZZ, Ning WB, Tao LJ: Fluorofenidone inhibits TGF-beta1 induced CTGF via MAPK pathways in mouse mesangial cells. Pharmazie 2009;64:680-684.

-25 Brenner BM, Cooper ME: Effects of Losartan on Renal and Cardiovascular Outcomes in Patients with Type 2 Diabetes and Nephropathy. N Engl J Med 2001;345:861-869.

-26 Chabrashvili T, Kitiyakara C, Blau J, Karber A, Aslam S, Welch WJ, Wilcox CS: Effects of ANG II type 1 and 2 receptors on oxidative stress, renal NADPH oxidase, and SOD expression. Am J Physiol Regul Integr Comp Physiol 2003;285:R117-R124.

27 Xiong X, Mei W, Xie Y, Liu J, Lu M, Peng X, Yang C, Zhang X, Xie M, Luo R, Yuan X, Huang L, Wu L, Qin J, Peng Y, Jia X, Hu G, Tang D, Tao L: Fluorofenidone Offers Improved Renoprotection at Early Interventions during the Course of Diabetic Nephropathy in db-db Mice via Multiple Pathways. PLos One 2014;27:e111242. 